Machado Chaviano, Esther Lidia. Integración y diseño del producto turístico. Aplicado a la región central del destino Cuba. GeoGraphos. [En línea]. Alicante: Universidad de Alicante, 15 de enero de 2013, vol. 4, nº 35, p. 69-92. [ISSN: 2173-1276].

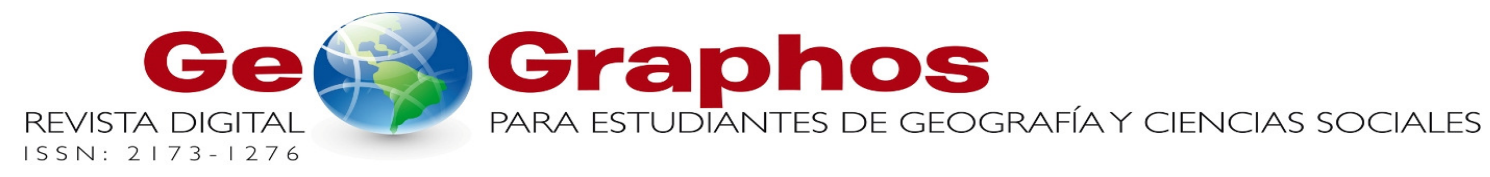

$<$ http://web.ua.es/revista-geographos-giecryal >

Año 2013

\title{
INTEGRACIÓN Y DISEÑO DEL PRODUCTO TURÍSTICO. APLICADO A LA REGIÓN CENTRAL DEL DESTINO CUBA
}

\author{
Esther Lidia Machado Chaviano \\ Centro de Estudios Turísticos de la Facultad de Ingeniería Industrial y Turismo \\ Universidad Central "Marta Abreu" de las Villas (Santa Clara, Villa Clara, Cuba) \\ Correo electrónico: esthermc@uclv.edu.cu
}

Recibido: 20 de julio de 2012. Devuelto para revisión: 10 de agosto de 2012.

Aceptado: 15 de enero de 2013

\section{RESUMEN}

Los destinos turísticos han tenido que incorporar nuevos conceptos en su gestión, debido a la globalización de los mercados y de la actividad turística en particular, lo que ha provocado un incremento en su competencia, en aras de despertar interés en el turista potencial y posicionarse en el lugar seleccionado para sus próximas vacaciones. Lo cual obliga al modelo de gestión tradicional a evolucionar, y mejoran así la competitividad del destino y los resultados económicos. La comercialización de los destinos debe perseguir la consolidación y el crecimiento, bajo una filosofía de marketing, y contribuir de forma sostenida, equilibrada y creciente a su desarrollo. La presente investigación propone, sobre la base de las tendencias actuales, un procedimiento científicamente fundamentado que logra diseñar nuevos productos turísticos integrados, permitiendo una estrategia de integración en la gestión del destino, y validan el mismo mediante su aplicación práctica en el diseño de productos sometiéndolo al juicio de expertos.

Palabras clave: Destinos, gestión, integración, producto turístico. 


\title{
INTEGRATION AND DESIGN OF TOURISM PRODUCTS. APPLICATION TO THE CENTRAL REGION OF CUBA DESTINY
}

\begin{abstract}
The tourist destinations have had to incorporate new concepts in their administration, due to the globalization of the markets in general and of the tourist activity in particular, what has caused an increment in their competition level, for the sake of to wake up interest in the potential tourist and to be positioned in the place selected for their next vacations. These elements are inserted in the administration process, they force to the pattern of traditional administration to evolve, and they improve this way the competitiveness of the destination and the economic results. The commercialization of the destinations should pursue the consolidation and the growth, under a marketing philosophy, and to contribute in a sustained way, balanced and growing to its development. In such a sense the present investigation proposes, on the base of the current tendencies of the tourist consumption, a scientifically based procedure that it is able to design new integrated tourist products that allow an integration strategy in the commercial administration of the destination, and they validate the same one by means of its practical application in the design of products subjecting it in the opinion of experts.
\end{abstract}

Key words: Destinations, administration, integration, tourist product.

\section{DESIGN E INTEGRAÇÃO DO PRODUTO TURÍSTICO. APLICAÇÃO PARA A REGIÃO CENTRAL DO DESTINO CUBA}

\section{RESUMO}

Os destinos turísticos tiveram que incorporar novos conceitos em suas administrações devido à globalização dos mercados em geral e da atividade turística em particular, o que causou um incremento em seus níveis de competição a fim de causar interesse no turista potencial e ser selecionado para as próximas férias. Estes elementos são inseridos no processo de administração, forçam o padrão de administração tradicional a evoluir e melhoram a competitividade do destino e os resultados econômicos. A comercialização dos destinos deveria procurar a consolidação e o crescimento, segundo uma filosofia de marketing, e contribuir de um modo contínuo, equilibrado e crescente ao seu desenvolvimento. Neste sentido, o presente estudo propõe, na base das tendências atuais do consumo turístico, um procedimento cientificamente baseado que pode projetar novos produtos turísticos integrados que permitam uma estratégia de integração na administração comercial do destino, e eles validam o mesmo por meio de sua aplicação prática no planejamento de produtos submetendo-o à avaliação de especialistas.

Palavras chave: Destinos, administração, integração, produto turístico.

\section{INTRODUCCIÓN}

La actividad turística es objeto de estudio de muchos especialistas, debido al suficiente impacto que provoca cambios en las economías, los entornos y las sociedades (Cooper, 2007). 
No obstante, los impactos positivos del turismo desde el punto de vista económico no deben sobrevalorarse, sino estudiarse, e incluso analizar las limitantes que surgen en el propio proceso con vistas a elevar sus resultados.

En la contemporaneidad, donde el desarrollo tecnológico provoca cambios significativos en la economía y la sociedad, el producto turístico adquiere un carácter más dinámico y activo; no es suficiente el hecho de mostrar algo, sino que además se hace necesario trasmitir una experiencia, criterios coincidentes en autores como Ávila y Barrado (2005), Kotler (2006), y Machado y Hernández (2008; 2009).

Por otro lado, el marketing, ha evolucionado a la par que el comportamiento de la sociedad, imponiéndose como filosofía en el mundo de los negocios provocando una nueva forma de trabajo: marketing colaborativo, de acuerdo con Peñarroya (2005), Kotler (2006) y Machado (2007); de tal modo cambia el papel de la entidad, y se pasa de dirigir y controlar al cliente, a conectar y colaborar con él.

La propuesta de integrar a la entidad, el cliente y la red de colaboradores, es una de las tendencias más significativas del nuevo marketing colaborativo (Bicné et al, 2000; Beltrán et al, 2002; Peñarroya, 2005; Ávila y Barrado, 2005; Kotler, 2006; Machado y Hernández, 2008).

De tal modo se hace evidente la necesidad de reformular las estrategias, lo cual conlleva que los procesos tradicionales de desarrollo transiten de una manera general de planteamientos a la de un marco de intervención más complejo, en el cual sea obligado atender a la totalidad del destino turístico (Peñarroya, 2005; Ávila y Barrado, 2005; Kotler, 2006; Hernández, 2006). A pesar de que los autores antes mencionados consideran la necesidad de integración del producto turístico en un destino, no abordan en sus obras los elementos procedimentales que deben caracterizar esta integración desde el momento en que se crea el producto turístico.

Con el fin de contribuir al desarrollo de destinos turísticos complejos, sostenibles y con capacidad para ganar cuota de mercado en el competitivo entorno actual, la presente investigación analiza cómo se producen los cambios en la oferta y la demanda turísticas, y proyecta cómo deberían evolucionar los conceptos y procedimientos de diseños de productos turísticos en estas condiciones, aplicando las mismas al caso Destino Cuba.

\section{LA PERSPECTIVA DEL MARKETING COLABORATIVO Y SU CONTRIBUCIÓN AL DISEÑO DE PRODUCTOS TURÍSTICOS INTEGRADOS}

La actividad turística provoca impactos sustanciales en las economías, los entornos y las sociedades, de los países que la desarrollan; por lo que para abordar estos temas se requiere del análisis teórico de varios elementos, es por ello que el objetivo del mismo está dado en fijar la investigación dentro del conjunto de conocimientos que comprende temáticas relacionadas con los destinos y el producto turístico, los cuales permitan orientar los pasos de forma adecuada para su diseño, a través del análisis y la interpretación del problema desde un plano tanto teórico como práctico. 


\section{La actividad turística, a partir de la evolución de la demanda y los destinos turísticos}

La humanidad en su avance desarrolló formas de viajes; que en un principio se manifestaron como viajes con fines económicos o políticos, y posteriormente se sumaron otros intereses hasta llegar al viaje por placer en la sociedad capitalista.

Por lo que se establece una relación entre turismo, economía y sociedad, la cual se evidencia en el análisis de las definiciones aportadas por diferentes autores, tal es el caso de Hanziquer y Krapf (1942) y Society Tourism (1979); citados en Molina (1991), OMT (1983), Figuerola (1991), Dacosta (1993), Acerenza (1993), Smith (1994), Martín (2003), Nácher y García (2004); citado en Martín (2006), Rodríguez (2005) y por último el Colectivo de autores (2005); quienes coinciden en sus definiciones en que la actividad turística comprende el conjunto de actividades y relaciones que se establecen a partir del desplazamiento de un individuo fuera de su lugar de residencia habitual, y debe ser voluntaria, temporal y sin ánimos de lucro; concepción que en su generalidad es compartida por la autora.

Esta propia relación que se establece entre el turismo como actividad económica y las sociedades, ha provocado que el mismo transite por diferentes etapas que determinan su desarrollo; apreciándose su avance paulatino en las relaciones que se producen entre los diversos intereses (políticos, económicos, religiosos, curiosos e instructivos), Desarrollándose una etapa a partir 1950 de crecimiento intensivo de la actividad con un desarrollo masivo de la demanda y elevándose los índice de calidad del servicio que existían hasta el momento, criterio con el que coincide autores como Muñoz (2004), Normal (2004), Fernández (2006), Martín (2006) y Cooper (2007).

Este desarrollo masivo ha provocado la saturación de los mercados, con multitud de ofertas poco diferenciadas y fácilmente sustituibles, los que compiten vía precio y crean una dificultad para la rentabilidad social del turismo; pues los cambios manifiestos a nivel social y económicos del mundo inciden significativamente en la actividad, provocando cambios en el consumo y por consiguiente una evolución en la demanda turística

Asumiendo la autora la definición aportada por Milio (2004) en la que se considera la demanda turística como: turistas que de manera individual o colectiva disponen de tiempo libre, fuentes de financiamiento y necesidad de viajar; se desplazan fuera de su domicilio habitual, motivados por productos o servicios turísticos creados con el objetivo de satisfacer sus necesidades de ocio, recreo, descanso, esparcimiento, cultura o contacto con la naturaleza.

Se coincide con los juicios emitidos por los autores Perelló (2001), Serra (2003), Ávila y Barrado (2005), Cohen (2005), Martín (2006) y Machado y Hernández (2009) cuando plantean que los cambios en el estilo de vida de los individuos, están produciendo en la demanda turística actual transformaciones que se concentran en: turistas más experimentados, con un mayor nivel de exigencia en la calidad, quienes realizan una fragmentación de sus vacaciones en viajes más cortos y frecuentes, con intereses especiales, espontáneos, y fuertemente preocupados por el estado de conservación del entorno, así como preferencia por la organización del viaje.

Estas tendencias de interrelación con la oferta centran los cambios del turismo en cuatro ámbitos: calidad, en función del desarrollo y la organización de nuevas formas; consumidores como individuos autónomos, segmentados, con alta experiencia de viaje y en busca de nuevos beneficios; producto, a partir de la exigencia de nuevas formas de diseño, que permitan nuevas experiencias para el turista, con alto nivel de diversificación; entorno, su relación con el medio ambiente visto de forma integrada 
y con un alto nivel de conservación, lo que significa que los destinos se enfrenta ante el reto de satisfacer un cliente activo, diversificado, responsable y exigente en cuanto a la calidad.

Considerando, por tanto, que en la concepción de la oferta destino se debe tomar en consideración su forma de consumo. El criterio ofrecido por Bigné et al. (2000) resulta el más apropiado para estos propósitos, debido a que plantea al destino turístico como combinaciones de productos, que ofrecen una experiencia integrada a los turistas; constituye así un valor percibido que puede interpretarse subjetivamente por los consumidores en función de la forma en que organizan el viaje.

En este espacio en el cual tiene lugar la confluencia de agentes, iniciativas y experiencias, que acaban moldeando un lugar como atractivo para la atención de la demanda turística, se constituye toda una red de empresas especializadas en la satisfacción, que se identifica por producto global (Perelló, 2001; Martín, 2006).

Sin embargo, una fragmentación de la oferta en el destino, además de la falta de coherencia y coordinación entre los diferentes actores, conduce a una falta de definición en el producto global.

De tal modo la autora plantea que para lograr un desarrollo turístico equilibrado, será necesario actuar sobre el conjunto de lo que significa el destino y convertirse este en la experiencia en sí misma, con lo cual se logra el posicionamiento en la mente de los consumidores.

\section{Valoración del producto turístico desde su perspectiva de oferta y consumo.}

Varios estudiosos de la actividad turística (Medlik y Middleton, 1973 y Middleton et al., 1978; citados por Borja et al., 2002; Cárdenas, 1983; Cárdenas, 1991; Acerenza, 1993; Dacosta, 1993; Bordas, 1994; Middleton, 1994; Novo, 1997; Rodríguez y Coll, 2000; Borja et al., 2002; Armadans, 2002; Boullón, 2002; Serra, 2003; Salinas, 2003; Valls, 2004; Milio, 2004; González, 2006; Soto y Lima, 2009) ofrecen definiciones sobre producto turístico. Al realizar el análisis de estas se pueden visualizar las particularidades y especificidades de las definiciones del producto turístico, así como la evolución que han tenido las mismas, al incorporarle elementos de marketing, que actualmente inciden en la conceptualización y el posicionamiento que alcanza un producto turístico en el mercado.

No obstante la autora considera que si bien las definiciones de producto turístico han evolucionado y se han incorporado nuevos elementos en función del consumo, aún su tratamiento en función de la integración a los restantes elementos a considerar por la actividad turística, resulta insuficiente para lograr la satisfacción de las necesidades del cliente actual y superar además sus expectativas.

El desarrollo de productos en un destino se concibe fundamentalmente a través de dos vías (Perelló, 2001):

- Planificación física y proyectada de inversiones, la cual suele ser responsabilidad de la administración local, y generalmente está vinculada con la preparación de planes de uso del suelo, y la construcción de infraestructuras de acceso, transportes y telecomunicaciones, de forma que proporcionan suministros básicos tales como electricidad o agua potable, para lo que se requiere de la participación de equipos multidisciplinarios formados por arquitectos, economistas, expertos de turismo y de mercado, sociólogos, entre otros;

- La segunda vía de desarrollo de productos se concibe a través del diseño de productos comerciales, sobre la base de la oferta real de atractivos, equipamientos y servicios, de 
forma que adapte el producto existente a las nuevas condiciones de la demanda, mejore la imagen del destino y logre la atracción de nuevos segmentos de mercado.

Con relación al desarrollo de productos básicamente se realiza a través de la investigación de mercado y se sustenta en los conceptos y técnicas de marketing que debe desarrollar el destino, por lo que resulta necesario el análisis de algunos de los procedimientos existentes en la literatura para su concepción

El análisis de los procedimientos que para estos efectos han propuesto autores tales como: Schnard, (1990), Santesmases (1993), Kotler (1996), Nieto y LLamanzaresar (1995), Mazur (2002), Martín (2003), Serra (2003), Medina y Santamarina (2004) y Grande (2005), permiten concluir: las etapas de generación de ideas y tamizado de las mismas, test de producto y test de mercado, son etapas coincidentes en todos los casos; la posición que ocupa la etapa de análisis del mercado difiere de ser el primer paso, o un paso intermedio, o se consideran las necesidades del mercado en el momento de su presentación a través del test de mercado; Tanto el test de producto, como su lanzamiento y puesta a prueba son elementos coincidentes en el $77 \%$ de los procedimientos analizados; en el $100 \%$ de los procedimientos analizados solo participa el creador del producto, es decir, su futuro explotador; se considera que los procedimientos analizados están diseñados para responder a modalidades muy puntuales dentro de la demanda turística, lo cual limita la vinculación en un mismo producto de varias modalidades, a partir de la generación de ideas sin previamente definir el segmento de mercado a trabajar y sus necesidades, en los procedimientos analizados no se tiene en cuenta la necesidad de establecer en aquellos recursos, tanto culturales como naturales, que constituyan patrimonio la interpretación del mismo, de forma tal que no se distorsione su valor. Lo cual significa que aún resulta insuficientes la adaptación a las nuevas tendencias que exige el mercado, para lo cual se hace necesario incorporar elementos relacionados: la participación, la integración, e interpretación que permitan una mayor coordinación de todos los actores del producto turístico y a su vez de la integración de los atractivos turísticos de un destino.

\section{Situación actual de la actividad turística en Cuba}

El auge que esta la actividad turística ha alcanzado en el destino Cuba, en función del potencial de recursos naturales y las características socioculturales del país, la convierten en una esfera fundamental de la economía cubana, tanto por el volumen de ingresos que genera, como por su efecto dinamizador sobre otras actividades sociales y económicas.

Cuba ha sido destino y escenario de ese continuo fluir y refluir de visitantes; de tal modo el surgimiento de la actividad en el país y su desarrollo han estado en estrecha correspondencia con su historia económica y político-social; criterio que comparten autores como Salinas y Estévez (1996), Colectivo de autores (2005), Fernández (2006) y Estévez et al (2007).

El país consolida la política de desarrollo turístico en los años noventa, con un incremento sostenido de los indicadores económicos. En esta década ocurre la última reestructuración, y en 1994 se crea el Ministerio de Turismo (Mintur), con el objetivo principal de establecer las políticas a seguir en este sector (Salinas y Estévez, 1996; Quintana et al, 2005; Martín, 2006; Estévez et al, 2007).

El destino Cuba, además del turismo de sol y playa, continúa trabajando por diversificar sus productos y desarrollar otras ofertas que incluyan como atractivo la cultura, la naturaleza y la náutica, 
para continuar siendo un turismo de paz, salud y seguridad, y un multidestino en sí mismo (Vázquez, $2005 ; 2006)$.

Cuba, por su parte, cuenta con condiciones naturales que satisfacen las expectativas de un gran número de modalidades, planteamiento con el cual coinciden Cárdenas (1995), Boullón (1998), Perelló (2001), Beltrán et al, (2002), Caballero, Korstanje (2006), Rodríguez (2006), Machado (2007) y Morales (2007). En relación con las playas (elemento dimensionador por excelencia del segmento turístico más sobresaliente en la actualidad), se plantea que existen más de 300 con parámetros de longitud, ancho de franja de sol, calidad de la arena y pendiente, que satisfacen las expectativas del turismo internacional. Además, el territorio nacional cuenta con riqueza de su flora, fauna y diversidad de paisajes, los cuales constituyen un patrimonio de envergadura. De acuerdo con el criterio de especialistas, Cuba ocupa el $5^{\text {to }}$ lugar en el planeta en cuanto al número de endémicos de su flora vascular natural (Instituto de Planificación Física, 1997).Existen otros potenciales importantes en el destino, entre los cuales pueden citarse los histórico-culturales, socioeconómicos, e infraestructurales.

$\mathrm{Al}$ igual que otros destinos turísticos del mundo, Cuba se enfrenta a este nuevo reto de la demanda turística, por lo que realizar el análisis del desarrollo que ha tenido esta actividad y el estado actual del mismo, permitirá determinar las premisas necesarias para garantizar los niveles de competitividad que exige hoy el mercado internacional.

Estudios del Instituto Nacional de Investigaciones Económicas (2010), plantean que las causas fundamentales de este comportamiento se deben en lo externo, a la crisis económica, las agresiones del gobierno norteamericano, el incremento del turismo interior por Europa, la competencia en el Caribe y América Central, el auge de la modalidad de cruceros, regiones emergentes como Europa del Este, Túnez, Turquía, la situación económica-política mundial y factores ambientales; lo cual unido a insuficientes conexiones aéreas, deficientes campañas publicitarias e insuficiente información en el destino, limitado desarrollo inversionista, apreciación del CUC y problemas de calidad del producto, han sido elementos que han influido en los resultados antes mencionados; sin embargo, la autora considera que esta situación no solo se debe a esto; además influyen la falta de adaptación del producto turístico cubano a las demandas actuales del mercado internacional, lo cual ha conducido al Mintur a definir dentro de sus objetivos estratégicos para el período, el diseñar y concretar una comercialización más eficiente del producto turístico, incrementar y diversificar una oferta turística más competitiva, entre otras (Mintur, 2009).

En los epígrafes antes abordado en la investigación se ha demostrado que el producto turístico influye en la satisfacción de la demanda actual, por lo cual resulta importante la aplicación de una filosofía de marketing al sector turístico cubano; por lo que la autora coincide con los criterios abordados por Hernández et al (2004), cuando refieren la importancia, que adquiere en las condiciones del socialismo actual del país, de aplicar estas técnicas y herramientas para el logro de la eficiencia en la producción y los servicios, en función del uso racional de los recursos y en la satisfacción de las necesidad, los deseos y las expectativas del cliente no con fines de lucro sino de elevación de la calidad de vida de la población.

En análisis previo de la región central del destino Cuba se aprecio la ausencia de un producto global del destino, por lo que, para determinar de forma más precisa la situación comercial de la región la autora propone aplicar el procedimiento para auditorias de marketing en entidades turísticas cubanas de Machado (2008b), arribando a las conclusiones siguientes: 
Planificación de las operaciones: las estrategias trazadas a corto, mediano y largo plazos, se realizan por cadena o grupo que administra sin integrar a todo el destino en función del posicionamiento que se desea alcanzar; se realizan pocos estudios de la demanda y en los pocos casos en que se realizan no son elementos de partida en la planificación de la gestión comercial

Control: se detectaron deficiencias en el sistema de control de las acciones de la comercialización, que permitan medir la efectividad de las decisiones de marketing; por otro lado se puede apreciar una escasa coordinación entre los factores que interactúan en cada uno de los destinos, operando cada uno de forma independiente en alcanzar sus objetivos económicos.

Estado actual de la oferta: existe poca diversificación del producto turístico; se cuenta con recursos que dan la posibilidad de proponer ofertas distintivas que permitan en los productos originalidad y autenticidad, elementos que constituyen uno de los principales atractivos que espera un turista, sin embargo se considera que los productos son monótonos y repetitivos, y ocasionan una estadía del turista muy corta; además de que la oferta tiene varios años de diseño y no se ha renovado en su presentación.

\section{PROCEDIMIENTO PARA EL DISEÑO DE PRODUCTOS TURÍSTICOS INTEGRADOS}

En este epígrafe se pretende brindar un procedimiento con carácter estructural, participativo, flexible y dinámico, para el diseño de productos turísticos integrados adaptados a las condiciones reales de la región central del destino Cuba, el cual tiene en consideración las interacciones no solo del producto, sino de los destinos, y permitirá el desarrollo de estos bajo las nuevas necesidades del mercado turístico actual y las características de la gestión de productos turísticos.

\section{Sistema de gestión integrada de destinos turísticos}

Se evidenció, hasta el momento la necesidad de trabajar de conjunto en los destinos turísticos, porque en la práctica constituyen un todo único provisto de una lógica interna e interrelaciones que requieren una coherencia en la concepción de su desarrollo, como instrumento esencial que facilita la comercialización eficaz de cualquier tipo de producto-servicio.

La puesta en práctica de modelos de desarrollo de carácter integrado, permitirá a los diferentes sectores y subsectores económicos avanzar en un mismo sentido, facilitar el mantenimiento y la conservación del medio físico-natural y cultural, y de esta forma lograr el ordenamiento territorial de los espacios con potencial turístico.

El sistema de gestión integrada del destino lo conforman dos dimensiones, una interna, la cual representa el soporte de la actividad turística, armónicamente integrado al turista, y como resultado de esta integración se obtendrá una segunda dimensión, lográndose la creación de sistemas de calidad y de gestión en función de la sostenibilidad de los productos.

En la actualidad existe la necesidad de que los nuevos productos turísticos del destino, respondan al mismo carácter integrador, en cuyo diseño deben tenerse en cuenta los aspectos antes mencionados, por lo cual constituye un error asumir el producto turístico como la suma de infraestructuras, servicios, empresas, tecnologías y recursos humanos. 


\section{El producto turístico integrado como concepto acorde a las condiciones actuales}

Una vez explicada la importancia de una gestión integrada del destino turístico, queda demostrada la necesidad de reevaluar a partir de los cambios que a nivel de consumo se están presentando, por lo que resulta imprescindible que los productos turísticos a crear incorporen el propio carácter integrador del destino.

La autora considera que se deben incluir en la definición de producto turístico integrado los principios de la experiencia y de la participación de la nueva sociedad, los cuales deben estar contenidos en ella y no concebirla como un conglomerado de atributos tangibles e intangibles, esto conlleva al trabajo del producto desde una perspectiva de integración.

De acuerdo con lo antes expuesto la autora desarrolla la propuesta en la que define producto turístico integrado como: la mezcla de atractivos, con facilidades y accesibilidad, estrechamente vinculados a los recursos humanos y empresariales, donde el espacio de cooperación entre los actores implicados se desarrolle bajo un carácter participativo, manteniendo un vínculo permanente entre los mismos, de forma tal que se logre una experiencia única al visitante (Machado y Hernández, 2008; 2009; 2010).

Este desarrollo, sobre la base del producto turístico integrado, depende de la capacidad para establecer y además mantener redes de colaboración e intercambio entre los diferentes integrantes y prestatarios que forman parte del destino. La creación de esta red de colaboración implica un comportamiento cooperativo y participativo entre entidades, agentes y organizaciones que se encuentran vinculados por relaciones económicas, geográficas, sociales y culturales en el destino.

Se concluye así que la política de desarrollo de productos turísticos integrados debe ser concebida sobre la base de su integración al destino, como un proceso creativo, desde el nacimiento de la idea hasta su puesta en marcha, orientado a satisfacer exigencias de mercados y segmentos específicos, y el procedimiento por el cual se diseñen los productos un trabajo artístico y original. Como resultado se debe conseguir la satisfacción de la demanda, pero sobre la base de una diferenciación que proporcione rentabilidad económica, social y ambiental al destino.

\section{Principales premisas para la aplicación del procedimiento propuesto en el diseño de productos turísticos integrados}

Tomando en cuenta que el producto turístico debe adecuarse a las condiciones actuales que exige el consumidor turístico, donde se logre no solo satisfacer las necesidades de los clientes que nos visitan, sino además superar sus expectativas (Machado y Hernández, 2008; 2009; 2010); se determina como premisa básica la participación de los entes implicados en el destino, a partir de la creación de un equipo de trabajo.

Vista la participación como proceso activo encaminado a transformar las relaciones de poder y esta alcanza distintos niveles y diferentes formas de expresión (Borda, 1991; citados por Gianoten y Wit, 2007).

Este equipo trabajará sobre la base de interrelaciones de cooperación y colaboración, para lo cual se requerirá de un ente gestor, que en cada localidad debe ser, de acuerdo con los requerimientos del instrumento propuesto, el gobierno local según sea el caso, provincial o municipal; sin embargo, 
desde la normativa legal el proyecto de Ley de Agencias de Viajes en la República de Cuba, establece en su Capítulo II artículo 3 a estas como las únicas autorizadas en el territorio nacional a diseñar, organizar y vender productos turísticos, por lo cual el ente gestor en la aplicación del procedimiento propuesto será la AA.VV. local o nacional según sea el caso.

La integración propuesta contribuirá a la creación de un producto que sea una experiencia a vivir y no un simple viaje contemplativo, y constituya una herramienta de aprendizaje (Machado y Hernández, 2009, 2010).

Otra de las premisas de aplicación del procedimiento consiste en la correcta interpretación del patrimonio tangible e intangible que se utilice en el producto a diseñar, por lo que las instituciones encargadas de conservar y gestionar el patrimonio son parte imprescindible del equipo de diseño.

\section{Propuesta de procedimiento para el diseño, sobre bases participativas, de productos turísticos integrados}

El procedimiento que se propone tiene por objetivo lograr una ventaja competitiva sobre la base del diseño de productos turísticos integrados, toma en consideración toda una serie de recursos históricos, culturales y naturales, que podrían incorporarse al mismo, sin perder la esencia de la visita del cliente y constituye una experiencia a vivir más que un simple viaje contemplativo.

El procedimiento consta de 9 etapas y 30 actividades. Una primera etapa organizativa; la segunda etapa de diseño constituye el nivel analítico o de diagnóstico; la tercera etapa aborda toda la actividad de búsqueda y selección de ideas a desarrollar; en las etapas cuarta y quinta se definen los atributos a partir de los requerimientos del mercado y se diseña el producto; la etapa sexta desarrolla el resto de las variables dependientes del marketing, precio, distribución y comunicación; la etapa séptima establece el presupuesto de gastos necesarios para el desarrollo del producto y el estimado de ingresos que por concepto de la comercialización de este se obtengan, se desarrolla a continuación una etapa octava de seguimiento y control; la última y novena etapa se reconoce como de retroalimentación, y es la encargada de retroceder a cualquiera de las etapas anteriores en el momento que se requiera. El orden en que deben realizarse las etapas y actividades se presenta en forma de esquema simplificado en la figura 1. 
Figura 1. Esquema simplificado del procedimiento para el diseño de productos turísticos en la región central del Destino Cuba

\begin{tabular}{|c|c|c|c|c|c|c|}
\hline $\begin{array}{c}\text { Etapa I } \\
\text { Organizativa }\end{array}$ & Etapa II Analítica & $\begin{array}{c}\text { Etapa III Generación de } \\
\text { ideas } \\
\text { Etapa IV Atributos } \\
\text { detallados } \\
\text { Etapa V Diseño de } \\
\text { productos }\end{array}$ & $\begin{array}{c}\text { Etapa VI } \\
\text { Estratégica }\end{array}$ & $\begin{array}{c}\text { Etapa VII } \\
\text { Previsión de } \\
\text { Gastos e ingresos }\end{array}$ & $\begin{array}{c}\text { Etapa VIII } \\
\text { Control y } \\
\text { seguimiento }\end{array}$ & $\begin{array}{c}\text { Etapa IX } \\
\text { Retroali- } \\
\text { mentación }\end{array}$ \\
\hline
\end{tabular}

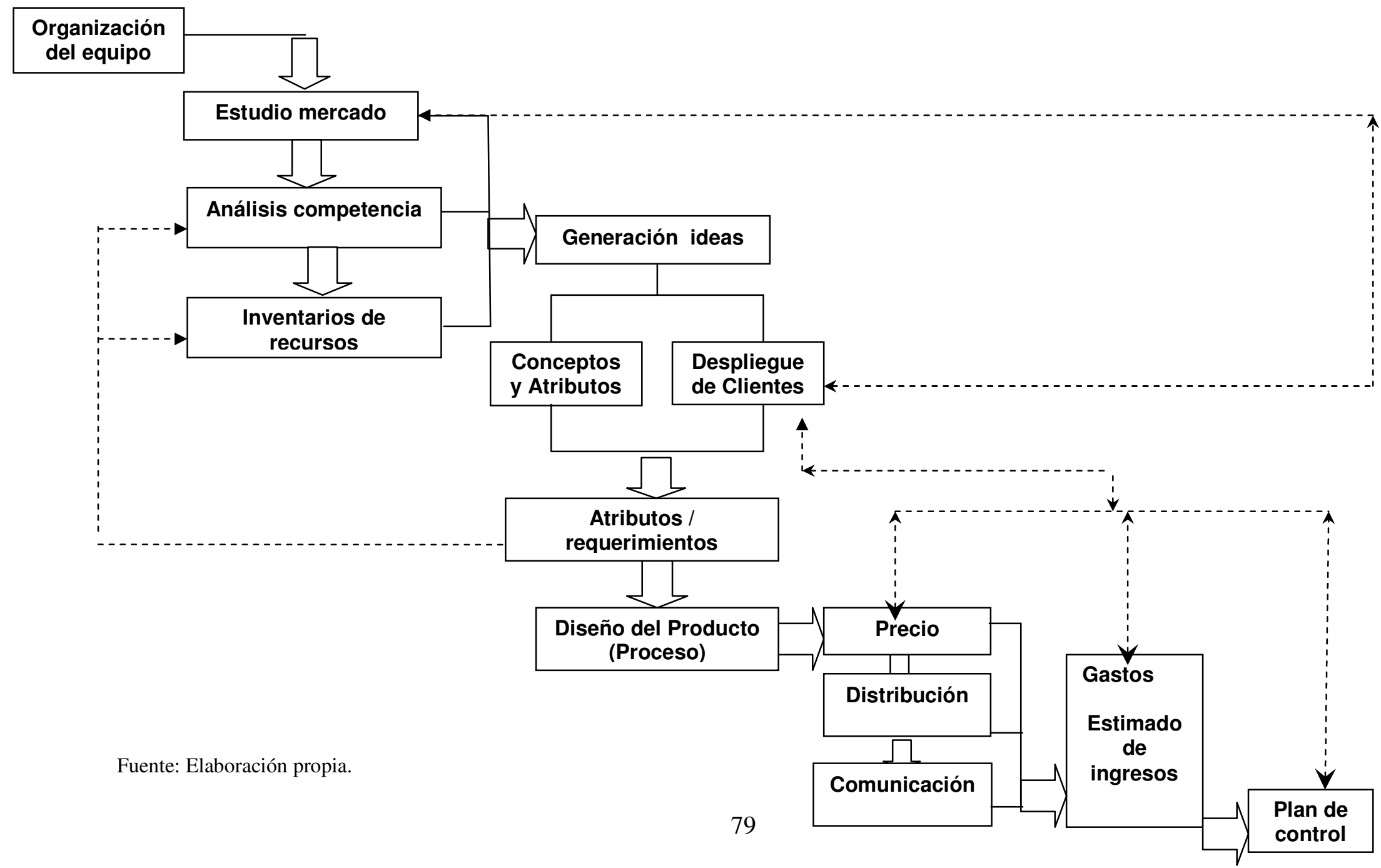




\section{Etapa I: Organizativa}

Esta es la primera etapa del procedimiento y es una de las más importantes, pues de ella depende en gran medida el avance del proceso de planificación, los objetivos y las estrategias que se definan. Es la etapa en la que se determina el nivel organizativo de trabajo que deberá emplear el equipo de diseño, y constituye los cimientos del producto.

La actividad número uno será la definición, por parte del equipo, de los objetivos de diseño, y la actividad número dos está constituida por la confección y aprobación del cronograma de trabajo, el cual deberá comprender todas las actividades que incluye el diseño del producto, así define la frecuencia de las reuniones de trabajo y la responsabilidad de cada miembro y enmarca en el tiempo el cumplimiento de las mismas.

\section{Etapa II: Analítica}

En la segunda etapa se diagnostica la situación actual en cuanto a demandas del mercado, análisis de la competencia e inventario de recursos turísticos con los que se dispone para el diseño del producto. Esta etapa comienza con la segmentación del mercado, la cual constituye la número tres y se realiza sobre la base de los criterios propuestos por Serra (2003).

Continúa la etapa con la selección de los segmentos de mercados claves; para esto la autora selecciona la tabla de segmentos de clientes, propuesta por Mazur (2002), la cual permite agrupar por características comunes, tanto demográficas como motivacionales, la segmentación anteriormente realizada.

Una vez segmentado el mercado y seleccionados de ellos los mercados claves, se deberá seleccionar el mercado meta, a partir de retomar los objetivos definidos por el equipo de diseño, y los segmentos de mercados claves; se establecen valores por cada miembro del equipo, se continúa con un estudio del mismo, a través de la ejecución de una investigación de mercado, que tome en consideración los tres tipos de requerimientos existentes: normales, esperados, y deleite (Kano et al., 1984).

La etapa II continúa con la actividad número cuatro, análisis de la competencia, para lo cual se propone la combinación de las propuestas realizadas por Acerenza y ESEM; se caracteriza la competencia a partir de los criterios definidos por Acerenza (2004), posteriormente se identifica cuáles de los competidores se encuentran en una categoría superior, y cuáles se encuentran en una categoría inferior.

Concluye la etapa con la actividad número cinco, análisis de los recursos turísticos disponibles, condición fundamental para el desarrollo del trabajo de diseño.

\section{Etapa III: Búsqueda y selección de ideas}

Esta etapa comienza con la actividad número seis, la cual consiste en la búsqueda de ideas; este proceso innovador debe realizarse sobre bases creativas; esta actividad se desarrolla mediante la aplicación de técnicas que tienen por objetivo el desarrollo de capacidades de innovación y originalidad en los individuos de una organización. La autora propone utilizar el diagrama de Causa y Efecto, desarrollado por Ishikawa en 1943 (Sociedad Latinoamericana para la Calidad, 2000), con 
el propósito de obtención de ideas.

Una vez que se obtienen las posibles ideas a trabajar, se debe pasar a su cribado; para ello, se toma en consideración el análisis de riesgos según la clasificación que ofrecen Zorrilla et al. (2007). Concluye esta actividad con la realización de un trabajo en grupo en el cual cada miembro del equipo de diseño otorgará valores de ocurrencia de cada uno de ellos.

Etapa IV: Definición de atributos del producto

Una vez que se selecciona la idea a desarrollar por el equipo de diseño, se debe proceder a su descomposición, a través de las dimensiones de producto turístico, tomando en cuenta los tres (3) niveles que establece Serra (2003) para un producto turístico: básico, tangible y aumentado. De esta forma quedan definidos los requerimientos del producto en sus tres niveles.

Una vez identificados todos los atributos del producto se debe pasar, mediante la utilización de técnicas de búsqueda de consenso como la votación ponderada y la comparación apareada, a la selección de aquellos atributos que constituyen la base del producto, los cuales no deberán ser cambiados bajo ninguna de las alternativas posibles del producto; al resto de los atributos que pudieran ser modificados se les realizará una valoración de posibles sustituciones, lo cual constituye la actividad número once.

\section{Etapa V: Diseño del producto}

Descompuestos en atributos-características los niveles del producto, relacionados directamente con los requerimientos del mercado, se procederá a la actividad número doce, que consiste en relacionar cada uno de ellos a los recursos turísticos, anteriormente inventariados.

Posteriormente se describen de forma detallada los atributos en ofertas y/o servicios, a continuación se realiza la matriz atributos detallados-requerimientos.

La siguiente actividad número dieciséis consiste en desplegar acciones concretas que se deberán desarrollar. Para dar cumplimiento al atributo detallado se utiliza la técnica de las $5 \mathrm{~W}+2 \mathrm{H}$ propuesta por Action Group (2009), la cual permite a partir del proceso de respuestas a siete (7) preguntas básicas (Qué, Quién, Cuándo, Dónde, Cuánto, Cómo y Por qué) listar de forma precisa y exacta qué hacer en cada momento del desarrollo del producto.

A partir de este desglose detallado de acciones se pasará a la actividad número diecisiete, diseño del proceso, que consiste en la definición de las características de los procesos claves del producto; recomendando, además, la construcción de un mapa de procesos, que una vez concluida las actividades concebidas, el equipo consulte a expertos en cada uno de los procesos.

\section{Etapa VI: Estratégica}

Es la fase superior del diseño de producto; permite detallar en estrategias y acciones concretas los pasos a seguir para llevar el producto diseñado al mercado meta. En la misma se definen las 
directrices generales del producto, para lo cual se toman como base los riesgos de la idea desarrollada, definidos en la actividad ocho.

En esta etapa se realiza la elaboración de la ficha de costo del producto y se determinan la accesibilidad del producto al consumidor final; se caracterizan los TTOO y las AAVV que operan en el mercado meta, y se define la imagen del producto diseñado.

Etapa VII: Previsión de gastos e ingresos

Una vez concluida la etapa estratégica, se debe tratar de estimar los recursos financieros que se requiere para su puesta en marcha, y además medirse la eficiencia del plan desde el punto de vista económico; para ello se ejecuta la actividad número veintidós, la cual parte de la previsión de las inversiones necesarias para llevar a cabo las acciones propuestas y la secuencia de gastos según los programas de trabajo y tiempos aplicados, u otros análisis económicos financieros que se estime necesario.

\section{Etapa VIII: Control y seguimiento}

El control es una importante etapa en el diseño del producto, de hecho si no se establecen los mecanismos de control adecuados, hay que esperar a que termine la ejecución de todas las acciones planificadas para comprobar si se ha logrado el objetivo marcado en el diseño del producto, pues se considera ésta una reacción tardía para cambiar el curso de las cosas. Es por ello que en este procedimiento se propone utilizar como mecanismo el control de rentabilidad y eficiencia del producto turístico, a través de la propuesta de control que realiza Kotler (1996), la cual permite conocer el grado de cumplimiento del producto a medida que se van ejecutando las acciones definidas y se van encaminando las estrategias. Con este control se pretende detectar los posibles fallos y desviaciones a tiempo, y prever con anticipación para poder aplicar soluciones y medidas correctoras con inmediatez.

\section{Etapa IX: Retroalimentación}

Es necesario supervisar constantemente la retroalimentación de los clientes y el personal involucrado. Cuando aparecen nuevos mercados y posibilidades se deberá retornar a aquella fase del procedimiento que se necesite, o por el contrario el propio desarrollo del producto determinará su evolución en el ciclo de vida, para lo cual la autora recomienda las siguientes estrategias a partir del ciclo de vida del producto: estrategias nivel crecimiento, estrategias nivel desarrollo, estrategias nivel declive.

\section{VALIDACIÓN DEL PROCEDIMIENTO PROPUESTO MEDIANTE SU APLICACIÓN EN EL DISEÑO DE NUEVOS PRODUCTOS TURÍSTICOS EN EL DESTINO CUBA}

Teniendo en cuenta los resultados investigativos obtenidos se propone la comprobación y validez del mismo a través de la aplicación práctica de este procedimiento para el diseño de productos turísticos, a partir de la consecución de las diferentes etapas que se plantean y el empleo de las herramientas de 
análisis necesarias para su desarrollo exitoso, en las AAVV receptivas representadas en el centro del país.

\section{Aplicación integral del procedimiento propuesto en el diseño del producto turístico integrado "Ruta Guerrillera"}

El producto que se presenta a continuación fue concebido inicialmente en su diseño como un producto territorial, que abarcaba los territorios de Sancti Spíritus y Villa Clara, dado lo atractivo del mismo se solicitó (por el Grupo Cubanacán S.A.) extender el mismo a todo el país, por lo que la autora considera significativo presentar, la totalidad del producto en su carácter nacional.

\section{Cumplimiento de premisas para aplicar el procedimiento}

Se identifican los principales agentes, se determina el nivel de implicación de cada uno, y se define como miembros del equipo de diseño: Comercial Delegación Cubanacán Centro, Comercial Agencia de Viajes Cubanacán Centro, Especialista en producto Grupo Cubanacán, Especialista comercial de Flora y Fauna, Especialista comercial de Ecotur Nacional, Historiador en Villa Clara.

En sesiones del equipo deberán participar además como miembros del mismo: funcionarios de Cultura, Patrimonio y delegados del Ministerio de la Agricultura de las localidades implicadas.

Etapa I: Organización del equipo de diseño.

Actividad número uno, el equipo de diseño define los objetivos de trabajo y el plan de trabajo, el cronograma de encuentros y el compromiso de resultados en el tiempo.

\section{Etapa II: Analítica}

La puesta en práctica de la actividad número tres, toma como referencia los estudios de mercado realizados por la Agencia Viajes Cubanacán (Grupo Cubanacán, 2003), en la que se obtiene la segmentación de los principales mercados con los que opera la agencia: parejas canadienses, familias canadienses, parejas alemanas, familias alemanas, hombres españoles, grupos de italianos, grupos de Francia y parejas de la tercera edad de Reino Unido (UK).

Continúa la etapa con la selección, así se toman en consideración las principales insatisfacciones y características de los segmentos anteriormente descritos, con vistas a la determinación de los mercados claves; para ello el equipo de diseño realiza el análisis de las tendencias de los principales mercados (investigación realizada por el Grupo Cubanacán, 2003) e incorpora además otros dos segmentos de mercados potenciales, estudiantes argentinos y chilenos. De este análisis se obtiene como resultado de mercados claves: parejas canadienses, grupos de italianos, parejas de Reino Unido y estudiantes argentinos y chilenos, a continuación se seleccionan los mercados metas, para ello se aplica la matriz, objetivos de diseño $v s$ mercados claves, la cual da como resultado que constituye la primera prioridad los estudiantes de Argentina y Chile, los cuales por su homogeneidad, el equipo decide considerarlos para el diseño con el segmento de mercado de estudiantes latinoamericanos; en 
segundo lugar las parejas del mercado UK; y en tercer lugar el segmento de grupos de italianos, a través de una investigación de mercado se obtienen los requerimientos por segmentos.

A continuación se desarrolla la actividad número cuatro, análisis de la competencia, en la que se realiza una valoración de las ofertas de la misma, sobre la base de los criterios definidos por Acerenza (2004) sobre las características del producto ofrecido, precios públicos, ingresos turísticos y los turistas/días atendidos por producto.

Este análisis permite determinar como competidor superior de la Agencia Viajes Cubanacán, a Havanatur y Cubatur, debido al nivel de operaciones que desarrollan en gran parte del territorio nacional.

Culmina la etapa II con la actividad número cinco, en la cual se realiza el inventario de recursos naturales, históricos, culturales e infraestructura disponible, en los territorios donde no tenían fuerte operación las AAVV competidoras, los cuales resultaron ser: Granma, Las Tunas, Ciego de Ávila, Sancti Spíritus y Villa Clara.

\section{Etapa III: Búsqueda y selección de ideas}

Esta etapa se comienza con la valoración del equipo de diseño de las necesidades insatisfechas de los segmentos meta, y se realiza una tormenta de ideas para buscar posibles soluciones; se manejaron varias ideas las cuales fueron sometidas a un análisis de pre-factibilidad y posibles riesgos para cada una de ellas, seleccionando la siguiente: Idea 1: Desarrollar un programa nacional para un turismo de aventura, que se conforme a partir del recorrido del Che en el proceso revolucionario cubano.

Etapa IV: Definición de atributos del producto

Se descompone la idea a desarrollar en niveles de producto turístico (básico, tangible y aumentado) a partir de la traducción en un servicio siguiendo la idea seleccionada.

A tenor del análisis del equipo, se determinan los requerimientos coincidentes entre los segmentos de mercado parejas UK y grupo de italianos, por lo que estos quedan establecidos como segmento de aventura; se definieron, por tanto, los atributos del producto en sus tres niveles, luego a través del consenso del equipo de diseño se seleccionan aquellos atributos que constituyen la base del producto, los cuales no deberán ser cambiados bajo ninguna de las alternativas posibles de producto: ruta temática, de intercambio con la naturaleza del país, siguiendo como guía: personalidades, ambientes, hechos y escenarios históricos-naturales con un alto reconocimiento internacional, a través de la modalidad de aventuras, en el que las experiencias del grupo de visitantes, y el intercambio con la comunidad, contribuya a generar una imagen de autenticidad, cubanía y sostenibilidad del producto.

\section{Etapa V: Diseño del producto.}

Esta etapa comienza con la asociación, por parte del equipo, de los atributos generales con el inventario de recursos, ya evaluados, de esta forma se obtienen como resultado las posibles actividades a realizar. 
Se retoma la técnica de generación de idea para detallar los atributos, los cuales son aprobados por el equipo bajo consenso de cada uno de sus miembros.

Atributos detallados:

1. Intercambio con la naturaleza, con fuerte componente histórico como atributo básico del producto, el cual debe contar con el reconocimiento a nivel nacional e internacional.

2. Desarrollo de la modalidad de aventuras como forma de presentación y penetración de este producto en los diferentes mercados.

3. Construcción de esquemas generales, desde donde saldrán las posibles combinaciones que puedan ser adecuadas a los requerimientos de cada segmento.

4. Aprovechamiento de la infraestructura existente que posibilitará el montaje de una base de operaciones flexible y móvil, la cual permita una protección a los costos y el uso de rutas y recursos no convencionales, en aras de garantizar la competitividad del producto.

5. Servicios no convencionales que permitan la integración y participación de la mayoría de los Grupos Empresariales existentes en el Mintur.

6. Participación e interactividad de los clientes con el producto, en escenarios previamente seleccionados.

7. Intercambio y vida en comunidades rurales.

8. Contribuirá a la preservación, desarrollo, y promoción del patrimonio histórico- natural nacional.

A partir de los elementos analizados se da paso al proceso de construcción de la ruta, se detallan las acciones a desarrollar en tiempo real y se define cada uno de los elementos que interactúan en el producto, y por ende se determinan de todos los factores que intervienen en el mismo, así como los medios que se necesitan.

\section{Etapa VI: Estratégica}

El equipo de diseño decide introducir el producto en el mercado turístico internacional; así enfatizan, a través de la comunicación, las ventajas del producto y evalúan el posible canal de distribución a utilizar; todo esto sustentado en estrategias y acciones.

Etapa VII: Previsión de gastos e ingresos

Se continúa con la estimación del presupuesto del producto, para lo que se confecciona su ficha de costo, y se define un precio máximo y un precio mínimo del producto; ambos precios no incluyen la boletería aérea, estableciéndose un margen de utilidad entre los prestatarios involucrados y la agencia de un $5 \%$, en una primera etapa del producto.

Una vez concebido el producto se presenta en Ferias y Convenciones turísticas tanto nacionales como internacionales y se aprecia interés por parte de las AAVV de los mercados metas, lo cual permite establecer un cálculo de la demanda a partir de la consulta al canal de venta y establecer supuestos de operación para el producto en sus primeros años de operación. 
Se realiza el cálculo del VAN, para una tasa de descuento del $13 \%$, según establece el Banco Nacional de la República de Cuba; así como el cálculo de la TIR y del período de recuperación de la inversión, obteniéndose los resultados siguientes:

VAN 8544

TIR $41 \%$

NPER 2 años aproximadamente

NPER = Períodos en los que se recupera la inversión inicial del proyecto.

De esta forma se demuestra la factibilidad del producto diseñado.

\section{Principales resultados de la aplicación del procedimiento propuesto en el diseño de otros productos turísticos integrados}

Con el objetivo de continuar la evaluación en las Agencias de Viajes radicadas en la región central del destino Cuba, respecto a la aplicación del procedimiento participativo para el diseño de productos turísticos propuesto, se diseñan varios productos:

Producto "Knowing Santa María":el equipo de diseño se apoya, en el alto valor natural y estéticopaisajístico de Destino Cayos de Villa Clara, utiliza la modalidad de turismo de naturaleza como forma de presentación y penetración en los mercados meta, potenciando la participación y el intercambio con este ecosistema; el recorrido se realizará siguiendo una vieja leyenda de los antiguos pobladores de la región, con recreación en la contemplación de la flora, la fauna y la historia.

Producto "Todo Alas": basado en el estudio realizado por especialistas de la migración de las aves a esta región, tanto provenientes del hemisferio norte como del sur, se propone el presente producto el cual tiene como atributo básico desarrollar en el cliente actividades de auto-guiaje, a partir de la entrega de materiales auxiliares que recogen mapas de propuestas de recorrido, inventario de aves con la información requerida de cada una de ellas; el cliente deberá identificar la observación de la misma a partir de la toma de fotografía y llenado de formulario, el cual será competitivo entre ellos.

La Agencia de Viajes Gaviotatur de conjunto con la Marina Gaviota Cayos de Villa Clara, identifica una demanda insatisfecha en el mercado de Reino Unido que visita el destino en realizar viajes de descubrimiento, de manera especial la modalidad de vida a bordo, razón por la cual se constituye un equipo de diseño que logra crear el producto "En busca del tesoro de Jack Rackham", tomando como partida el gran número de cayos y cayuelos que existen en la costa centro norte del país con diversidad de flora y fauna, y retomando, además, la historia de corsarios y piratas de la región, se construye un recorrido en el que el cliente es el principal protagonista, de forma tal que se sienta re-descubridor de la zona y de sus valores, siguiendo la historia del pirata Jack Rackham.

Producto "Calidad de vida con biofuncionales" con el objetivo de diversificar la oferta dentro del Destino Cayos de Villa Clara, se decide, aprovechando la infraestructura complementaria existente en el mismo (Centro de Salud y Belleza) y la experiencia, preparación y oferta de biofuncionales del Centro de Biaoactivos Químicos de la Universidad Central "Marta Abreu" de Las Villas, conformar un equipo de diseño con el objetivo de crear un producto que consiste en un paquete de servicios destinados al diagnóstico, prevención y tratamiento de secuelas de enfermedades degenerativas y otras disfunciones, en pos de trabajar en lograr el mejoramiento de la calidad de vida de los clientes; a 
partir de su interacción con el producto, el cliente selecciona el programa de tratamientos y terapias, en dependencia de sus necesidades y expectativas, siendo algunos servicios de necesario cumplimiento. Los tratamientos se basan en el uso de productos con propiedades biofuncionales, en su gran mayoría de origen nacional, a partir de un diagnóstico realizado empleando un software creado con este propósito, exámenes físicos, y análisis adicionales en caso necesario. Estos tratamientos para combatir secuelas generadas por enfermedades de gran impacto, se complementan con servicios recomendados por los especialistas, relacionados con ejercicios, masajes, máscaras, terapias y bailes de distintos tipos. Se toman como escenarios para ello, diversos espacios que reúnan las condiciones requeridas para desarrollar las actividades, incluyendo áreas de masajes, gimnasio, SPA (Centro de Salud por Agua) y espacios naturales como la playa o parques; de esta forma el cliente puede beneficiar del producto paralelo a su estancia vacacional.

El Destino Cienfuegos, con un fuerte inventario de recursos náuticos e históricos-culturales ha mostrado una tendencia al decrecimiento en arribo de clientes en los últimos 4 años, con características específicas de los clientes que viajan al destino, quienes prefieren moverse libremente y de forma independiente, por tal motivo la Agencia de Viajes Cubanacán de este territorio decide conformar un equipo de diseño. El estudio de mercado detectó una demanda insatisfecha de los clientes del destino en realizar paseos por la bahía de forma dinámica e instructiva, elementos en los que se basó el equipo para diseñar el producto turístico "Travesías de Leyendas", el cual consiste en una opcional, auto-guiada que propicia el desarrollo de las actividades náuticas en vínculo armonioso con las más exóticas leyendas sobre la ocupación y el asentamiento en Cienfuegos de los siboneyes, así como de españoles y franceses en la ciudad, el cual presenta como atributo básico el disfrute del esplendor panorámico de la bahía y la ciudad, a diferentes horas del día, ya que se encuentra ubicada en una posición privilegiada desde el mar.

Tomando en cuenta la tendencia internacional de la actividad turística y la política del Destino Cuba de desarrollar el turismo de naturaleza, se identifica en el mercado de Reino Unido que visita el país la necesidad de intercambiar con la naturaleza cubana, creándose el equipo de diseño en el Destino Cienfuegos integrado por la Agencia Havanatur Tour \& Travel Centro, Flora y Fauna y CITMA; así se diseña el producto "Caminos de Cima a Sima", asentándose en la diversidad de ecosistemas de esta región, este propone realizar un recorrido que, como su nombre indica, realiza un desplazamiento del vértice de la región hasta la parte más baja de la costa, contemplando e intercambiando con la variedad de flora, fauna y paisajes, donde se desarrollan actividades de autoguiaje del cliente.

Producto "El Valle: entre azúcar y haciendas", la presente opcional se diseña en el Destino Trinidad y basa su desarrollo en clientes interesados en conocer e intercambiar con la historia de la Trinidad de Cuba y el Valle de los Ingenios (considerados ambos patrimonio de la humanidad); el mismo permite al turista absorber las distintas sensaciones que se vivieron en el siglo XIX en el Valle de los Ingenios, haciéndolo sentir como un personaje de ese siglo; además participa en los procesos manuales y fabriles de la producción azucarera en el Valle, e intercambia con las comunidades locales cercanas.

\section{CONCLUSIONES}

1. Los rasgos fundamentales en las tendencias de la demanda turística y la incidencia de estas en la oferta actual y su comercialización, así como la necesidad de la elaboración e implementación de procedimientos para el diseño de productos turísticos integrados, debido a la no adaptación de los procedimientos existentes a la situación actual; los nuevos procedimientos deben incluir 
elementos de la participación, integración e interpretación de valores; como guía planificada, lógica, y estructurada de las diferentes variables del marketing.

2. A partir de la evaluación de la situación actual de la gestión de marketing en las entidades del sector turístico en la región central del Destino Cuba; se pudo constatar que en la mayoría de los casos existe un conjunto de problemas relacionados con la aplicación del marketing, lo cual provoca una desfavorable situación comercial, y marcada tendencia al decrecimiento de sus indicadores y problemas en su oferta.

3. Al considerar los resultados tanto positivos como negativos que hasta el momento se han obtenido como parte de la comercialización de productos turísticos, la autora propone un nivel de integración de los entes implicados en el destino, a través de un sistema donde los productos turísticos logren la combinación de los atractivos, con las facilidades y acceso de un destino; se define el producto turístico integrado y se propone un procedimiento para el diseño, el cual consta de 9 etapas y 30 actividades muy bien integradas, y logra adaptarse a las características de la nueva demanda turística, en las condiciones actuales de la región central del destino Cuba.

4. Mediante la aplicación del procedimiento participativo para el diseño de productos turísticos integrados en la región central del destino Cuba se obtiene un total de (9) productos turísticos integrados. Se realiza la validación del procedimiento propuesto mediante el juicio de expertos, se puede afirmar que si se diseñan productos turísticos integrados partiendo de un procedimiento con carácter participativo, flexible y dinámico, adaptado al entorno de la región central del destino Cuba, es posible contribuir a la diversificación de la oferta de la región y a un mejor aprovechamiento de los recursos turísticos disponibles a través de la venta de los nuevos productos diseñados; por lo que queda demostrada la hipótesis de la investigación.

\section{BIBLIOGRAFÍA}

ACERENZA, M. Promoción Turística: un enfoque metodológico. Ciudad México: Editorial Trillas, 1993.

ACERENZA, M. Gestión de Marketing de Destinos Turísticos en el Ambiente competitivo actual en Aportes y Transferencias. Mar del Plata. Universidad Nacional de Mar del Plata, 2004, Año 7, vol. II, p. 43-56.

Action Group. Técnicas para el análisis de problemas: 5W +2". Mejora continua, desarrollo organizacional [En Línea]. 2009. <http://www.actiongroup.com.ar/dowload/5w2h.pdf $>$. [21 de marzo de 2009].

ARMADANS, I. Actividades de ocio turístico y personas mayores. Tesis Doctoral dirigida por Pol i Urrutia y Sopena. Barcelona: Universidad de Barcelona, 2002. p. 18-39.

ÁVILA, R. y BARRADO, D. Nuevas Tendencias en el Desarrollo de Destinos Turísticos: Marcos Conceptuales y operativos para su Planificación y Gestión. Cuadernos de Turismo [En Línea]. 2005, $\mathrm{n}^{\mathrm{o}} 15$. <http://www.cuadernosdeturismo.com> . [8 de marzo de 2008]. 
BELTRÁN, L.; LÓPEZ, H. y GÓMEZ, J. Diseño y comercialización de Productos Turísticos. Tesis de Licenciatura. Colombia: Instituto Distrital de Turismo [En Línea]. 2002 $<$ http://www.bogotaturismo.gov.co/descargas/turismo/IDCT-DISENO.doc $>$. [7 de diciembre de 2008].

BIGNÉ, E.; FONT, X. y ANDREU, L. Marketing de Destinos Turísticos: Análisis y estrategias de desarrollo. Madrid: Editorial ESIC, 2000.

BORDAS, E. Convertir recursos turísticos en productos turísticos. Actas del II Simposio Internacional de Turismo. Barcelona: ESADE, 1994.

BORJA, L.; CASANOVA, A. y BOCH, R. El Consumidor Turístico. Madrid: Editorial ESIC, 2002.

BOULLÓN, R. Marketing Turístico: Una perspectiva desde la planificación. Buenos Aires: Colección Temas de Turismo, 1998.

CABALLERO, A. y PONS, R. Posicionamiento estratégico de productos turístico Monografías [En Línea]. 2004. <http://www.monografías.com/trabajos39/productos-turisticos/productos-

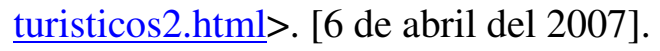

CÁRDENAS, F. Producto Turístico. Aplicación de la estadística y del muestreo para su diseño. Ciudad de México: Editorial Trillas, 1983.

CÁRDENAS, F. Mercadotecnia y productividad turística. Ciudad de México: Editorial Trillas, 1991.

CÁRDENAS, F. Producto Turístico. Ciudad de México: Editorial Trillas, 1995.

COHEN, E. Principales tendencias en el turismo contemporáne. Revista Política y Sociedad. Jerusalem: Department of Sociology and Anthropology. The Hebrew University of Jerusalem, 2005, vol. $42, \mathrm{n}^{\mathrm{o}} 1, \mathrm{p}$ 11-24.

Colectivo de Autores. Agencias de Viajes. Monografías [En Línea]. 2000. $<$ http://www.monografias.com/trabajos11/trabagenc/trabagenc.shtml-73k >. [19 de enero del 2009].

Colectivo de Autores. Material de apoyo sobre mercados emisores. Ciudad de la Habana: Universidad de La Habana, 2005.

COOPER, C. El turismo teoría y práctica. Madrid: Síntesis, 2007.

DACOSTA, J. Diccionario de Turismo y Hotelería. Caracas: Panapo, 1993.

ESEM-Auditorías de marketing. Material de apoyo Maestría Marketing y Gestión Comercial. Madrid: Escuela Superior de Estudios de Marketing de Madrid, 1999. p. 6-29.

FERNÁNDEZ, N. El turismo de frente al futuro. Texto docente. Camagüey: Escuela de Hotelería y Turismo Hermanos Gómez, 2006.

FIGUEROLA, M. Teoría económica del Turismo. Madrid: Alianza, 1991. 
GIANOTEN, V. y WIT, T. Un caso de autoorganización popular, Diccionario crítico de Ciencias Sociales [En Línea]. 2007. <http://www.ucm.es/info/eurotheo/diccionario.htm>. [15 de enero del 2007].

GRANDE, I. Marketing de los servicios. Madrid: ESIC, 2005.

GONZÁLEZ, J. Marketing. Investigación y Marketing [En Línea]. 2006. $<$ http://europa.sim.ucm.es/compludoc/AA?articuloId=534500\&donde=castellano $>$. [19 de abril de 2009].

Instituo de Planificación Física (IPF). Potencial Habitacional para el Desarrollo Ecoturístico de Cuba. Ciudad de la Habana: Departamento de Planeamiento Turístico, 1997. p 23.

Instituto Nacional de Investigaciones Económicas. Tendencias y Perspectiva de desarrollo del Turismo en Cuba. Conferencia impartida por Alfredo García Jiménez. Villa Clara: $2^{\mathrm{do}}$ Evento ECONDETUR, 2010.

KOTLER, P.; BOWEN, J. y MAKENS, J. Mercadotecnia para Hotelería y Turismo. Ciudad de México: Prentice-Hall Hispanoamericana, 1996.

KOTLER, P. El pulso entre Marketing y ventas: Cómo pasar del enfrentamiento a la colaboración. Revista Daemon Quest [En Línea]. 2005. <http://www.daemonquest.com>. [7 de abril de 2007].

KOTLER, P. La retención de un cliente leal. Revista Daemon Quest [En Línea]. 2006.

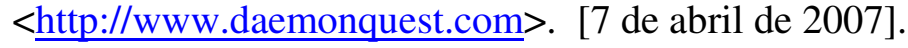

LEFCOVICH, M. Auditoría interna. Un enfoque sistémico y de mejora continúa [En Línea]. 2003. $<$ http://www.avizora.com>. [30 de septiembre de 2008].

MACHADO, E. y CUEVAS, O. Propuesta de procedimiento para el Diseño de Productos Turísticos Integrados en el Destino Cuba. Tesis en opción al grado de Máster dirigida por Dr. C. Carlos Cristóbal Martínez. Ciudad de la Habana: Facultad de Comunicaciones. Universidad de La Habana, 2007.

MACHADO, E. y HERNÁNDEZ, Y. Procedimiento para el diseño de productos Turísticos integrados en Cuba". Revista Teoría y Praxis, 2008 a, vol. 4, año 3.

MACHADO, E. y HERNÁNDEZ, Y. Diagnóstico y propuesta de procedimiento para la auditoría de gestión del marketing en entidades turísticas cubanas. Revista Gestión Turística, 2008 b, n 10, p. 4157.

MACHADO, E. y HERNÁNDEZ, Y. Del Turismo contemplativo al Turismo activo. Revista Periplo Sustentable, 2009, n 15 p 111-122.

MACHADO, E. y HERNÁNDEZ, Y. Procedimiento participativo para el diseño de un producto turístico integrado en el destino Cuba. Revista Retos Turísticos, 2010 a, vol 9, $\mathrm{n}^{\circ}$.1. ISSN: 1681-9713 
MACHADO E. y HERNÁNDEZ, Y. La actividad turística en Cuba análisis crítico de la aplicación del marketing en el sector empresarial. Revista Folletos Gerenciales. Ciudad de la Habana: Universidad de la Habana. Cuba, 2010 b, n 1 , año XIV.

MARTín, R. Manual de Teoría y Práctica del Turismo. Texto Docente. Ciudad de la Habana: Centro de Estudios Turísticos Universidad de La Habana [En Línea]. 2003. <http://intranet.uclv.edu.cu/fiit/cetur>. [3 de febrero de 2007].

MARTÍN, R. Principios, organización y práctica del turismo. Ciudad de la Habana: Centro de Estudios Turísticos. Universidad de la Habana, 2006.

MARX, C. El Capital Tomos I, II y III. Ciudad de la Habana: Editorial de Ciencias Sociales, 1973.

MAZUR, G. Despliegue de la Función de Calidad. Material del curso de Productos Turísticos. Ciudad de la Habana: Grupo Cubanacán, 2002.

MEDINA, N. y SANTAMARINA, J. Turismo de Naturaleza. Ciudad de la Habana: Ediciones Unión, 2004.

MIDDLETON, V. Marketing in Travel and Tourism. Londres: Butterworth, 1994.

MILIO, I. Diseño y comercialización de productos turísticos locales y regionales. Madrid: ThopsonParaninfo, 2004.

MOLINA, S. y RODRÍGUEZ, S. Planificación Integral del turismo. Un enfoque para Latinoamérica. Ciudad México:Trillas, 1991.

MORALES, C. Diseño de opcionales turísticas en las Agencias de Viajes de Villa Clara. Tesis en opción al grado de Máster dirigida por Dr. C. Carlos Cristóbal Martínez. Villa Clara: Universidad Central "Marta Abreu de Las Villas", 2007.

NIETO, A. y LLAMANZARES, O. Marketing Internacional. Madrid: Pirámide, 1995, p. 112-175.

NORMAL, A. La Industria Turística”. Traducción de Francisco Muñoz de Escalona. Murcia: Edición eumed.net [En Línea]. 2004. <http://www.eumed.net/cursecon/librería >. [15 de diciembre de 2007].

NOVO, G. Diccionario General de Turismo. Ciudad de México: Diana, 1997, p. 273.

Organización Mundial del Turismo. Clasificación Internacional propuesta por la OMT. Madrid: Ediciones OMT, 1983. p. 9.

PEÑARROYA, M. Philip Kotler los 10 principios del nuevo Marketing. Revista Daemon Quest [En Línea]. 2005. <http://www.daemonquest.com>. [10 de noviembre de 2007].

PERELLÓ, J. Desarrollo y promoción de productos turísticos. Curso de postgrado Universidad de La Habana. Ciudad de la Habana: Facultad de Estudios Turísticos [En Línea]. 2001. $<$ http://intranet.uclv.edu.cu/fiit/cetur/comercializacionturistica $>$. [8 de febrero de 2007]. 
QUINTANA, R. y FIGUEROLA, M. et al. Efectos y Futuros del Turismo en la economía cubana. Villa Clara: Intranet Universidad Central "Marta Abreu" de las Villas [En Línea]. 2005. $<$ http://intranet.uclv.edu.cu/fiit/cetur $>$. [ 20 de marzo de 2008].

RODRÍGUEZ, R. Gestión de Destinos Turísticos. Texto docente. Ciudad de la Habana: Centro de Estudios Turísticos Universidad de la Habana, 2005.

RODRÍGUEZ, R. Desarrollo de productos turísticos. Monografía. Ciudad de la Habana: Centro de Estudios Turísticos Universidad de la Habana [En Línea]. 2006.

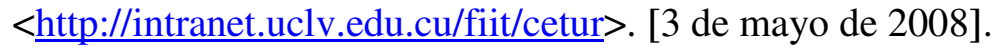

SALINAS, E. y ESTÉVEZ, R. Aspectos territoriales de la actividad turística en Cuba. Revista Estudios Geográficos, 1995, vol. 52, n 223, p. 327-350.

SALINAS, E. Geografía y Turismo. Aspectos territoriales del manejo y gestión del turismo. La Habana: Ediciones SI-MAR, 2003.

SANTESMASES, M. Marketing: conceptos y estrategias. 2a edición. Madrid: Pirámide, 1993.

SCHNARD, A. Nuevo producto. Bogotá: McGraw Hill, 1990.

SERRA, A. Marketing Turístico. Madrid: Pirámide, 2003.

SMITH, S. The Tourism Product. Annals of Tourism Research [En Línea]. 1994, vol. 21 $<$ http://www.sciencedirect.com/science?_ob=articleURL\&udi=B6V7Y-46BHY11-

F8\&_user $=10 \& \_c o v e r D a t e=12 \%>$. [14 de marzo del 2008].

Sociedad Latinoamericana para la Calidad. Diagrama Causa y Efecto [En Línea]. 2000.

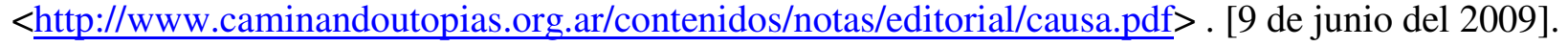

SOTO, J. y LIMA, E. Boletín Turístico. Perú [En Línea]. 2009. <http://www.boletinturistico.com/lexico/lexicop.htm> . [25 de abril del 2009].

VALLS, J. Gestión de destinos turísticos sostenibles. Madrid: Gestión 2000, 2004.

ZORRILLA, G.; ZAMORA, D. y ACOSTA, W. Dirección y Evaluación de Proyectos Turísticos. Libro de Texto Licenciatura en Turismo. Ciudad de la Habana: Universidad de la Habana, 2007.

(C) Copyright Esther Lidia Machado Chaviano, 2013.

(C) Copyright GeoGraphos, 2013.

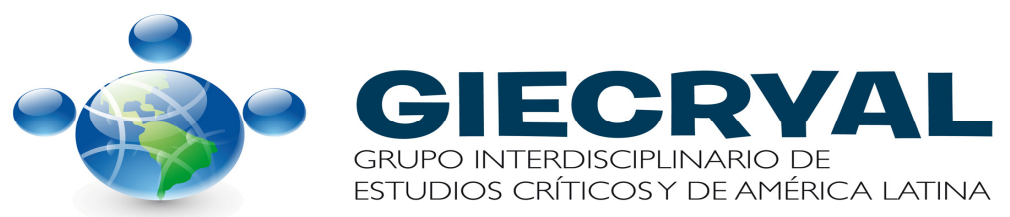

